\title{
Percepción de prácticas parentales en adolescentes tempranos y tardíos: Una perspectiva del desarrollo
}

Blanca Estela Barcelata Eguiarte y Vianey Gutiérrez Arenas

\section{Resumen}

La familia es fundamental en el desarrollo del ser humano. Las prácticas de crianza de los hijos durante la adolescencia deben cumplir con dos funciones medulares: brindarles seguridad y permitir el desarrollo de la autonomía. Esta investigación tuvo como objetivo responder a la pregunta: ¿Existen diferencias en la percepción de las prácticas parentales entre adolescentes tempranos y los tardíos y de acuerdo con el sexo? Participaron 357 adolescentes escolares de 13 a 18 años de la Ciudad de México (investigación financiada por PAPIIT IN305917), a quienes se les aplicó el EMBU-A para adolescentes mexicanos. Para el análisis de los datos se utilizó el programa SPSS 21. Los adolescentes tempranos perciben mayor calidez de ambos padres mientras que los adolescentes tardíos perciben mayor control parental y menor favoritismo. No obstante, en general los adolescentes perciben menor calidez y mayor rechazo del padre, y mayor favoritismo de ambos padres. 
Palabras clave: Desarrollo; adolescentes; prácticas de crianza; EMBU; padres.

\section{Introducción}

La adolescencia es una etapa crucial del desarrollo humano. Se distingue por cambios vertiginosos de índole física, psicológica y social (Musitu y Cava, 2003) y comprende aproximadamente de los 10 a los 20 años (UNICEF, 2011). La mayoría de los teóricos del desarrollo (p. ej., Sameroff, 2009; Sroufe, 2009) coincide en que la principal tarea es el logro de la identidad y la autonomía, lo cual involucra la interacción dinámica de diferentes sistemas, empezando por la familia. En este sentido, pueden existir diferentes trayectorias y resultados de la adolescencia en función de dicha interacción.

La adolescencia representa un periodo amplio del ciclo vital que tiende a ser dividido en etapas o subetapas que involucran marcadores biológicos y psicológicos que suelen variar de acuerdo con el contexto. Algunos investigadores (Casas y Ceñal, 2005; Spano, 2004) identifican tres etapas: 1) adolescencia temprana (10 a 13 años), caracterizada por un desarrollo físico acelerado, enfocado principalmente en la maduración de los órganos sexuales; 2) adolescencia media (14 a 17 años); los cambios físicos son mucho más lentos, mientras que el desarrollo de capacidades cognitivas, así como la adquisición paulatina de independencia y autonomía de la familia, alcanzan gran relevancia; y 3) la adolescencia tardía (17 a 20 años); se adquiere una mayor conciencia de sí mismo, del futuro y de las implicaciones de sus actos. La UNICEF (2011), por su parte, considera dos etapas: la adolescencia temprana, que comprende de los 10 a los 14 años, y la adolescencia tardía que va de los 15 a los 19 años. Estas dos etapas tienen algunas características distintivas que involucran cambios importantes en los procesos cognitivos, perceptuales, emocionales y relacionales, en especial con la familia.

Para muchas familias, la adolescencia de los hijos puede representar una etapa de crisis, debido a las grandes demandas de recursos de diversa índole (p. ej., económicos, instrumentales) por parte del adolescente hacia los padres (Padilla, Fajardo, 
Gutiérrez y Palma, 2007). La familia se enfrenta a un reajuste de los roles familiares para cumplir con la compleja tarea de encontrar un equilibrio entre dar a los adolescentes la independencia necesaria para su individuación y, al mismo tiempo, protegerlos de decisiones o comportamientos que pudieran ponerlos en riesgo (Minuchin, Nichols y Lee, 2011; Mitsu, Buelga, Lila y Cava, 2004). Por ello, la familia ejerce una influencia importante en su desarrollo integral, proporcionándoles seguridad y bienestar como agente socializador y trasmisor de valores a nivel intergeneracional (UNICEF, 2004). El apoyo emocional, instrumental, físico y/o económico de los padres es un factor clave de protección para los adolescentes (Estévez, Musitu y Herrero, 2005). Para brindar dicho apoyo, los padres realizan una serie de comportamientos denominados prácticas parentales o prácticas de crianza.

Las prácticas parentales, también denominadas prácticas de crianza, pueden entenderse como acciones que han sido aprendidas por los padres y que son puestas en práctica con la finalidad de educar y orientar a los hijos, para favorecer una adecuada integración social (Ramírez, 2005; Solís-Cámara y Díaz, 2007). Representan un conjunto de comportamientos dentro de un proceso interaccional padres-hijos en la cotidianeidad que puede estar caracterizada por el poder de los padres hacia los hijos y tienden a cambiar o a modificarse en función de la etapa del desarrollo (Aguirre, 2000; Andrade y Betancourt, 2012).

De acuerdo con Izzedin y Pachajoa (2009), la crianza de los hijos está mediada por tres aspectos principalmente: 1) las pautas de crianza (la normatividad cultural en torno a la crianza), 2) las prácticas de crianza (acciones concretas), y 3) las creencias acerca de la crianza (ideas personales en torno a la crianza). Las pautas de crianza y las creencias son ideas, prejuicios, conocimientos y normas que permiten al padre darle sentido a las prácticas de crianza que emprende. Diversos estudios han reportado los efectos que determinadas prácticas pueden generar en los adolescentes (Hernández, González, Bermúdez, Freyre y Alcázar-Olán, 2013; López-Rubio, Fernández-Parra, Vives-Montero y Rodríguez-García, 2012).

Las prácticas parentales positivas crean un contexto para la empatía, expresión de emociones, comunicación clara y abier- 
ta, con límites claros y disciplina, enfocadas en la resolución de conflictos y donde los hijos se sienten apoyados, por lo que están relacionadas con hijos más seguros, autónomos, con madurez emocional y habilidades de comunicación e interacción (Castillo, 2016; Cuervo, 2010). Por el contrario, las prácticas parentales negativas, que incluyen un estilo de comunicación autoritario, el uso de castigos constantes, una expresión negativa de las emociones, límites confusos o inconsistentes y, en general, control psicológico (Andrade y Betancourt, 2012; Hernández, 2015), se relacionan alteraciones emocionales y de conducta que pueden traducirse en problemas internalizados y/o externalizados (p. ej., expresar emociones, socializar, seguir normas y/o presencia de conductas antisociales).

A pesar de la importancia que tienen las prácticas parentales o de crianza durante la adolescencia, la mayoría de los estudios se enfocan en la infancia. Muchos de los estudios que evalúan la percepción de la crianza por parte de los adolescentes se enfocan en las diferencias por sexo, siendo relativamente escasos los que también exploran el efecto de la edad o subetapa de la adolescencia. Sin embargo, desde un punto de vista del desarrollo, la adolescencia representa un periodo amplio en el que los procesos cambian rápidamente de una subetapa a otra en función de la edad y el desarrollo alcanzado. Considerando esto, la pregunta general de este estudio fue: ¿Existen diferencias en la percepción de las prácticas parentales entre los adolescentes tempranos y los tardíos? Por lo tanto, el objetivo principal fue analizar la percepción de hombres y mujeres, adolescentes tempranos y tardíos, de las prácticas parentales.

\section{Método}

\section{Participantes}

Participaron de manera intencional, 357 adolescentes de escuelas públicas de nivel secundaria y preparatoria, distribuidas en la Zona Metropolitana de la Ciudad de México; con un rango de edad comprendido entre los 13 a 18 años ( $M_{\text {edad }}=15.30$; D.E.=1.60); de los cuales $54.34 \%$ fueron mujeres y $45.66 \%$ hombres. 


\section{Instrumentos}

Para evaluar las prácticas de crianza, se aplicó la prueba Egna Minnen Betraffande Uppfostran (EMBU-I) (Perris, Jacobsson, Lindström, Von Knorring y Perris, 1980); validada con adolescentes mexicanos por Márquez-Caraveo, Hernández, Aguilar, Pérez y Reyes (2007) y ajustada por Hernández (2015). Consta de 80 reactivos Likert de cuatro puntos: 1) No, o nunca, 2) Sí, algunas veces, 3) Sí, frecuentemente, y 4) Sí, casi siempre, distribuidos en cuatro factores: 1. Calidez ( $\alpha=.91)$ : Implica apoyo parental a través de conductas como aceptación, comunicación, orientación, búsqueda de recursos para beneficio del adolescente y respeto por su autonomía, a través de manifestaciones físicas y verbales; 2 . Rechazo ( $\alpha=.83)$ : Refiere a conductas de los padres como hostilidad, tratos desiguales, agresivos, indiferencia, negligencia, menosprecio, desaprobación, crítica, culpabilización; 3. Control ( $\alpha=.82)$ : Indaga conductas parentales como presión, inducción, chantaje, ejercer imposiciones mediante fuerza o amenaza, así como sobreprotección y monitoreo excesivo del comportamiento de los hijos; y 4. Favoritismo $(\alpha=.81)$ : Valora las conductas de los padres que conllevan distinciones entre los hijos y beneficios. El alfa total es de .91.

Para identificar algunas variables sociodemográficas, se aplicó la Cédula Sociodemográfica del Adolescente y su Familia (Barcelata, 2016). Es un autoinforme conformado por 16 reactivos que explora indicadores sociodemográficos del adolescente y su familia ( $\left.\alpha_{\text {ordinal }}=.76\right)$.

\section{Procedimientos}

Se realizó un estudio de campo transversal, ex post facto, multivariado (financiado por PAPIIT IN305719) de una sola muestra con un diseño $2 \times 2$. Se solicitó colaboración a las autoridades de escuelas públicas de la zona Metropolitana de la Ciudad de México. Se entregaron consentimientos informados a las autoridades de las escuelas y asentimientos garantizando la participación voluntaria y confidencial. Se consideraron adolescentes tempranos a los chicos entre 13 a 16 años que generalmente acuden a 
la escuela secundaria, y como adolescentes tardíos a los de 16 a 18 años que asisten regularmente al bachillerato o preparatoria. La aplicación se llevó a cabo de manera grupal, con la colaboración de tres a cuatro aplicadores y un supervisor. Las aplicaciones tuvieron una duración promedio de 60 minutos.

Con el uso SPSS 21, se realizaron análisis descriptivos, bivariados (ANOVA) y multivariados (MANOVA), con el objetivo de examinar diferencias por edad y sexo, así como efectos principales y de interacción de estas dos variables en la percepción de las prácticas de crianza.

\section{Resultados}

Características de los participantes

La distribución por edad y sexo de los 357 estudiantes se presenta en la Figura 1, de los cuales 53.8\% son estudiantes de nivel secundaria y $46.2 \%$ de nivel preparatoria. Los participantes de secundaria son en su mayoría (89\%) adolescentes tempranos (13 a 16 años), mientras que 93\% de los estudiantes de preparatoria o bachillerato son adolescentes tardíos (16 a 18 años).

Mujer Hombre

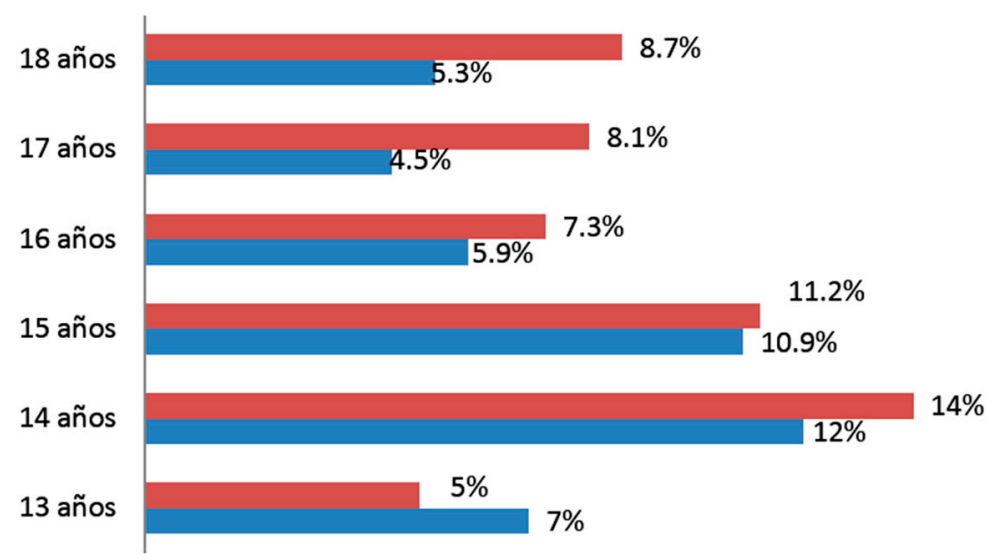

Figura 1. Distribución de la muestra por sexo y edad 
La Tabla 1, muestra las medias y desviación estándar de las dimensiones que componen el EMBU-A, comparándolas por sexo. En todas las dimensiones, tanto para la práctica parental como maternal, se puede encontrar una media mayor en el grupo de los hombres que en el de las mujeres, en ambos grupos de edad. Asimismo, se aprecia que en todos los grupos, la dimensión con mayor media es la Calidez, mientras que el Favoritismo muestra las puntuaciones más bajas. En ambos sexos, las medias de la práctica materna fueron superiores que las medias de la práctica paterna.

Tabla 1. Medias y desviaciones estándar de los análisis univariados de las prácticas de crianza materna y paterna por sexo

\begin{tabular}{|c|c|c|c|c|c|c|c|c|c|c|}
\hline \multirow[t]{3}{*}{ Factor } & \multicolumn{2}{|c|}{$\begin{array}{c}\text { Hombres } \\
n=163\end{array}$} & \multicolumn{2}{|c|}{$\begin{array}{c}\text { Mujeres } \\
n=194\end{array}$} & \multirow[t]{3}{*}{$\mathbf{F}$} & \multicolumn{2}{|c|}{$\begin{array}{c}\text { Hombres } \\
n=163\end{array}$} & \multicolumn{2}{|c|}{$\begin{array}{c}\text { Mujeres } \\
n=194\end{array}$} & \multirow[t]{3}{*}{$\mathbf{F}$} \\
\hline & \multicolumn{4}{|c|}{ Práctica paternal } & & \multicolumn{4}{|c|}{ Práctica maternal } & \\
\hline & $\mathbf{M}$ & D.E. & $\mathbf{M}$ & D.E. & & $M$ & D.E. & $\mathbf{M}$ & D.E. & \\
\hline Calidez & 2.49 & .671 & 2.38 & .746 & 1.41 & 2.81 & .542 & 2.72 & .607 & 3.37 \\
\hline Rechazo & 1.60 & .515 & 1.54 & .493 & 1.22 & 1.60 & .536 & 1.57 & .450 & .501 \\
\hline Control & 1.93 & .491 & 1.90 & .495 & .493 & 2.19 & .494 & 2.19 & .443 & .717 \\
\hline Favoritismo & 1.49 & .508 & 1.46 & .396 & .684 & 1.55 & .506 & 1.47 & .386 & $4.98^{*}$ \\
\hline
\end{tabular}

$\mathrm{M}=$ Media; $\mathrm{DE}=$ Desviación Estándar; F = Estadístico de prueba * $p<.05$

En la comparación de las medias entre grupos de edad (Tabla 2), se encontraron medias más altas en las dimensiones relacionadas con la práctica maternal. La dimensión de Calidez (tanto paterna como materna) obtuvo las medias más altas, en ambos grupos de edad, mientras que el Favoritismo (paterna y materna), las medias más bajas. 
Tabla 2. Medias y desviaciones estándar de los análisis univariados de las prácticas de crianza materna y paterna por edad

\begin{tabular}{|c|c|c|c|c|c|c|c|c|c|c|}
\hline \multirow[t]{3}{*}{ Factor } & \multicolumn{2}{|c|}{$\begin{array}{l}13-15 \\
n=210\end{array}$} & \multicolumn{2}{|c|}{$\begin{array}{c}16-18 \\
n=146\end{array}$} & \multirow[t]{3}{*}{$\mathbf{F}$} & \multicolumn{2}{|c|}{$\begin{array}{l}13-15 \\
n=210\end{array}$} & \multicolumn{2}{|c|}{$\begin{array}{c}16-18 \\
n=146\end{array}$} & \multirow[t]{3}{*}{$\mathbf{F}$} \\
\hline & \multicolumn{4}{|c|}{ Práctica paternal } & & \multicolumn{4}{|c|}{ Práctica maternal } & \\
\hline & M & D.E. & $\mathbf{M}$ & D.E. & & M & D.E. & M & D.E. & \\
\hline Calidez & 2.42 & .684 & 2.44 & .756 & .168 & 2.62 & .581 & 2.83 & .572 & $3.30^{*}$ \\
\hline Rechazo & 1.55 & .486 & 1.58 & .529 & .397 & 1.58 & .477 & 1.58 & .510 & .127 \\
\hline Control & 1.94 & .477 & 1.86 & .511 & $2.04^{*}$ & 2.08 & .462 & 2.22 & .472 & $2.398 *$ \\
\hline Favoritismo & 1.48 & .440 & 1.46 & .465 & .056 & 1.50 & .402 & 1.50 & .503 & .289 \\
\hline
\end{tabular}

$\mathrm{M}=$ Media; $\mathrm{DE}=$ Desviación Estándar; $\mathrm{F}=$ Estadístico de prueba, ${ }^{*} \mathrm{p}<.05$

La Tabla 3 permite identificar los datos por sexo y grupo de edad. Los resultados muestran que en ambos grupos de edad, las medias tanto para las prácticas paternas como maternas son mayores para las mujeres. Las medias tanto para los adolescentes tempranos como tardíos son superiores en las dimensiones de las prácticas maternas que paternas.

Tabla 3. Medias marginales y desviaciones estándar de los análisis multivariados de las prácticas de crianza materna y paterna por edad y sexo

\begin{tabular}{|c|c|c|c|c|c|c|c|c|c|}
\hline $\begin{array}{l}\text { Factores } \\
\text { Crianza }\end{array}$ & \multicolumn{4}{|c|}{$\begin{array}{l}\text { Adolescentes tempranos } \\
\text { 13-15 años } \\
\mathrm{N}=210\end{array}$} & \multicolumn{4}{|c|}{$\begin{array}{l}\text { Adolescentes tardíos } \\
\text { 16-18 años } \\
\mathrm{N}=146\end{array}$} & \multirow[t]{3}{*}{$\mathbf{F}$} \\
\hline & \multicolumn{2}{|c|}{$\begin{array}{l}\text { Hombres } \\
n=106\end{array}$} & \multicolumn{2}{|c|}{$\begin{array}{l}\text { Mujeres } \\
n=104\end{array}$} & \multicolumn{2}{|c|}{$\begin{array}{l}\text { Hombres } \\
n=56\end{array}$} & \multicolumn{2}{|c|}{$\begin{array}{l}\text { Mujeres } \\
n=90\end{array}$} & \\
\hline & $M$ & DE & $M$ & DE & $M$ & DE & $M$ & DE & \\
\hline \multicolumn{10}{|l|}{ Paterna } \\
\hline Calidez & 2.493 & .6881 & 2.346 & .6755 & 2.482 & .6500 & 2.421 & .8226 & .302 \\
\hline Rechazo & 1.584 & .4949 & 1.533 & .4783 & 1.642 & .5592 & 1.544 & .5129 & .183 \\
\hline Control & 1.959 & .4864 & 1.939 & .4708 & 1.881 & .4989 & 1.859 & .5216 & .300 \\
\hline Favoritismo & 1.483 & .4442 & 1.484 & .4409 & 1.513 & .6182 & 1.431 & .3404 & .700 \\
\hline
\end{tabular}




\begin{tabular}{|c|c|c|c|c|c|c|c|c|c|}
\hline \multirow[t]{3}{*}{$\begin{array}{l}\text { Factores } \\
\text { Crianza }\end{array}$} & \multicolumn{4}{|c|}{$\begin{array}{l}\text { Adolescentes tempranos } \\
\text { 13-15 años } \\
\mathrm{N}=210\end{array}$} & \multicolumn{4}{|c|}{$\begin{array}{l}\text { Adolescentes tardíos } \\
16-18 \text { años } \\
\mathrm{N}=146\end{array}$} & \multirow[t]{3}{*}{$F$} \\
\hline & \multicolumn{2}{|c|}{$\begin{array}{l}\text { Hombres } \\
n=106\end{array}$} & \multicolumn{2}{|c|}{$\begin{array}{l}\text { Mujeres } \\
n=104\end{array}$} & \multicolumn{2}{|c|}{$\begin{array}{l}\text { Hombres } \\
n=56\end{array}$} & \multicolumn{2}{|c|}{$\begin{array}{l}\text { Mujeres } \\
n=90\end{array}$} & \\
\hline & M & DE & $\mathbf{M}$ & DE & $M$ & DE & $\mathbf{M}$ & DE & \\
\hline \multicolumn{10}{|l|}{ Materna } \\
\hline Calidez & 2.797 & .5695 & 2.642 & .5854 & 2.841 & .4916 & 2.827 & 6199 & 1.26 \\
\hline Rechazo & 1.600 & .5092 & 1.578 & .4448 & 1.567 & .4959 & 1.568 & .4583 & .030 \\
\hline Control & 2.159 & .4778 & 2.193 & 4483 & 2.245 & .5225 & 2.194 & 4401 & .699 \\
\hline Favoritismo & 1.503 & .3944 & 1.516 & .4132 & 1.649 & .6624 & 1.520 & .3475 & $6.25^{*}$ \\
\hline
\end{tabular}

$\mathrm{M}=$ Media; $\mathrm{DE}=$ Desviación Estándar; $\mathrm{F}=$ Estadístico de prueba. ${ }^{*} \mathrm{p}<.05$

En relación con las prácticas paternas, en ambos sexos y en ambos grupos de edad, las medias más altas se ubican en la dimensión de Calidez, mientras que Favoritismo, fue la dimensión con medias inferiores. Lo mismo ocurre con las prácticas maternas en el grupo de Adolescentes tempranos (tanto hombres como mujeres) y con las mujeres del grupo de Adolescentes tardos; puntúan más alto en Calidez y más bajo en Favoritismo. Sin embargo, en el grupo de los hombres adolescentes tardíos las medias más bajas se ubican en la dimensión de Rechazo.

Los resultados del análisis multivariado de la varianza (MANOVA) indican efectos principales significativos de la edad $\left(F_{W_{i-}}\right.$ $\left.{ }_{\mathrm{k}}=22.436, p=.01 ; n=.53\right)$, del sexo $\left(F_{\text {wiks }}=22.305, p=.02, n=51\right.$. $)$ y de interacción del sexo*edad $\left(F_{\text {wilks }}=22.563, p .01, n=.56\right)$. Tanto la edad como el sexo son variables que tienen un efecto significativo en la percepción de los adolescentes, respecto a las prácticas de crianza de sus padres. Asimismo, las prácticas de crianza materna en todos los grupos (sexo y edad) tienen las medias más altas, en comparación con las prácticas paternas.

\section{Discusión}

El objetivo de este estudio fue analizar si existen diferencias en la percepción de las prácticas parentales entre adolescentes tempranos y los tardíos en función del sexo. Los resultados refle- 
jan que los adolescentes perciben mayor calidez, es decir, mayor afecto por parte de ambos padres. Esta percepción se relaciona probablemente con un desarrollo psicosocial positivo, un mayor bienestar emocional y una comunicación recíproca funcional con los padres (Andrade y Betancourt, 2012; Castillo, 2016; Hernández, 2015), sin embargo, en este estudio no se presentan datos sobre el nivel de bienestar o de adaptación de los adolescentes.

Respecto a las prácticas de control, los adolescentes presentan puntuaciones moderadas y bajas, lo que podría deberse a que se trata de una muestra escolar "normativa" cuyos padres no abusan del control, ni utilizan estrategias de presión o coercitivas (Ramírez, 2005). De manera similar a estudios previos (Frías, Fraijo y Tapia, 2012; Hernández, 2015), los adolescentes de esta muestra perciben menos prácticas parentales relacionadas con Rechazo y Favoritismo, lo cual podría estar reflejando prácticas parentales más equilibradas basadas en el acompañamiento (Chías y Zurita, 2009).

En cuanto al sexo, los resultados revelaron que tanto los hombres como las mujeres refieren mayor Calidez y menor Favoritismo de parte de ambos padres, sin embargo, los hombres perciben más Rechazo que las mujeres, especialmente del padre y particularmente los hombres de mayor edad. Esto pudiera deberse a factores culturales, como premisas y creencias culturales respecto a la educación y la "buena crianza" (Solís-Cámara y Díaz, 2007) que no fomentan interacciones activas, de apego, de comunicación abierta (Estévez et al., 2005; Izzedin y Pachajoa, 2009) de los padres hacia sus hijos varones y, en contraste, promueven más comportamientos que implican distancia física, aparente "indiferencia" y bajo involucramiento emocional, por lo que los padres tienden a ser más estrictos, lo que eventualmente puede aumentar la presencia de problemas en los hijos adolescentes (Hernández, 2015; López-Rubio et al., 2012; Márquez-Caraveo et al., 2007). No obstante, cabe señalar que en este estudio las puntuaciones en estos factores o dimensiones de las prácticas parentales fueron moderadas y por debajo de la media teórica.

Por otro lado, tanto hombres como mujeres perciben a las madres como más cálidas en comparación con los padres, lo que concuerda con los hallazgos encontrados por Oudhof, Rodríguez 
y Robles (2012). En cuanto al control conductual y psicológico, las madres son percibidas como más controladoras, en comparación con los padres tanto por los hombres como por las mujeres, sin diferencias estadísticas. Estos datos en conjunto sugieren que los papás tienden a no ejercer un adecuado uso del poder, de reconocimiento y de respeto de la autonomía de los hijos; además, de que tienden a ser más impositivos al establecer normas, reglas y límites, poco flexibles a la negociación (Minuchin et al., 2011; Romagnoli y Cortese, 2016).

En cuanto a la edad o etapa, no se encontraron diferencias significativas entre los adolescentes tempranos y tardíos en la percepción de todas las prácticas de crianza paternas y maternas. Sólo se aprecian diferencias en Calidez y Control, aunque el modelo general de interacción fue significativo para Favoritismo. Los datos indican que los adolescentes tardíos o de mayor edad perciben mayor calidez de la madre, aunque también mayor percepción de control tanto por los hombres como por las mujeres. Estos resultados coinciden tanto con los postulados teóricos (Minuchin et al., Sameroff, 2009; Sroufe, 2009) como con la evidencia empírica (Estévez et al., 2005; Musitu y Cava, 2003) que precisa que a medida que avanza la adolescencia hacia la adultez los adolescentes tienden a buscar mayor independencia y a autoafirmarse, por lo que perciben mayor control a sus padres (Leon et al., 2008; Mitsu et al., 2004; Spano, 2004).

En síntesis, hay que subrayar que los adolescentes tardíos o mayor edad perciben significativamente mayor control materno y menos prácticas paternas de calidez y de control, a pesar de que los hombres de menor edad tienden a reportar mayor control paterno. Por otro lado, los adolescentes en general perciben a las madres más controladoras, pero a la vez más cálidas, lo que parece reflejar que son las que están más al tanto de los hijos, más involucradas con su crianza y, al mismo tiempo, son más expresivas y cálidas, generando mayor percepción de apoyo, en especial en los hombres de mayor edad, lo que puede resultar positivo para los hijos (Estévez et al., 2005; Musitu y Cava, 2003). Por el contrario, los padres parecen más restrictivos, distantes y menos involucrados con la crianza de sus hijos, lo que nuevamente parece relacionarse con los patrones de interacción familiar basados 
en creencias más tradicionales, que no parecen haber cambiado con el tiempo (Minuchin et al., 2011; Solís-Cámara y Díaz, 2007).

Estos hallazgos confirman la necesidad de generar programas de intervención desde la escuela en la que se fomente la integración de los padres, usando estrategias más activas por parte de las autoridades escolares para convocar a los padres como una parte fundamental de los programas escolares. No obstante, se reconocen algunas limitaciones de este estudio como el hecho de que no se presentan resultados en cuanto a indicadores de salud mental, de bienestar psicológico o de adaptación positiva, por lo que sería recomendable que otros estudios incluyeran esta variable como evidencia del impacto de las prácticas en el bienestar integral de los adolescentes. Asimismo, sería importante explorar la estructura de la familia y su relación con las prácticas parentales, así como contrastar diferentes tipos de muestras de distintos contextos.

\section{Agradecimientos}

Este estudio fue realizado gracias al financiamiento de la DGAPA de la UNAM a través del proyecto PAPIIT IN305917. Asimismo, se agradece al psicólogo Guillermo Castillo de la Rosa por su colaboración en el armado de datos.

\section{Referencias}

Andrade, P. y Betancourt, D. (2012). Problemas emocionales y conductuales en niños: predictores desde la percepción de los padres y de los hijos. Acta de Investigación Psicológica, 2(2), 650-664.

Barcelata, B. (2016). Cédula Sociodemográfica del Adolescente y su Familia. Proyecto PAPIIT IN303514. DGAPA-UNAM, versión para investigación. México: FESZ, UNAM.

Casas, J. y Ceñal, M. (2005). Desarrollo del adolescente. Aspectos físicos, psicológicos y sociales. Pediatría Integral, IX(1), 20-24.

Castillo, G. (2016). Competencias socioafectivas y su relación con las prácticas de crianza en adolescentes. Tesis de Licencia- 
tura. FESZ, UNAM, México, D.F.

Chías, M. y Zurita, J. (2009). Emoción y arte con los niños. El arte de acompañar a los niños en su emoción. Madrid: Desclée de Brouwer.

Cuervo, A. (2010). Pautas de crianza y desarrollo socioafectivo en la infancia. Diversitas-Perspectivas en Psicología, 6(1), 111-121.

Estévez, E., Musitu, G. y Herrero, J. (2005). El rol de la comunicación familiar y del ajuste escolar en la salud mental del adolescente. Salud mental, 28(4), 81-89.

Frías, M., Fraijo, B. y Tapia, C. (2012). Prácticas de crianza y desarrollo afectivo y emocional de los niños. Psicumex, 2, 30-41.

Hernández, C. M. (2015). Prácticas parentales y su relación con la adaptación en la adolescencia. Tesis de Licenciatura. FESZ, UNAM, México, D.F.

Hernández, L., González, M., Bermúdez, G., Freyre, M. y Alcázar-Olán, R. (2013). Parental Practices Scale for Children. Revista Colombiana de Psicología, 22(1), 151-161.

Izzedin, R. y Pachajoa, A. (2009). Pautas, prácticas y creencias acerca de la crianza... ayer y hoy. Liberabit, 15(2), 109-115. León, D., Camacho, R., Valencia, M. D. y Rodríguez, A. R. (2008). Percepción de la función de sus familias por adolescentes de la enseñanza media superior. Revista Cubana de Pediatría, 80(3), 0-0.

López-Rubio, S., Fernández-Parra, A., Vives-Montero, M. y Rodríguez-García, O. (2012). Prácticas de crianza y problemas de conducta en niños de educación infantil dentro de un marco intercultural. Anales de Psicología, 28(1), 55-65.

Márquez-Caraveo, M., Hernández, L., Aguilar, J., Pérez, V. y Reyes, M. (2007). Datos psicométricos del EMBU-I "Mis memorias de crianza" como indicador de la percepción de crianza en una muestra de adolescentes de la Ciudad de México. Salud Mental, 30(2), 58-66.

Minuchin, S., Nichols, M. P. y Lee, W. Y. (2011). Evaluación de familias y parejas. Del síntoma al sistema. México: Paidós Mitsu, G., Buelga, S., Lula, M. y Cava, M. (2004). Familia y adolescencia. Un modelo de análisis e intervención psicosocial. Madrid: Síntesis. 
Musitu, G. y Cava, M. (2003). El rol del apoyo social en el ajuste de los adolescentes. Intervención Psicosocial, 12(2), 179-192.

Oudhof, H., Rodríguez, B. y Robles, E. (2012). La percepción de la crianza en padres, madres e hijos adolescentes pertenecientes al mismo núcleo familiar. Liberabit. Revista de Psicología, 18(1), 75-81.

Padilla, M., Fajardo, C., Gutiérrez, A. y Palma, D. (2007). Estrategias de afrontamiento de crisis causadas por desempleo en familias con hijos adolescentes en Bogotá. Acta Colombiana de Psicología, 10(2), 127-141.

Perris, C., Jacobson, L., Lindström, H., Knorring, L. y Perris, H. (1980). Development of anew inventory for assessing memories of parental rearing behavior. Acta Psychiatry Scandinavian, 61, 265-274.

Ramírez, M. A. (2005). Padres y desarrollo de los hijos. Prácticas de crianza. Estudios Pedagógicos, 31(2), 167-177.

Romagnoli, C. y Cortese, I. (2016). ¿Cómo la familia influye en el aprendizaje y rendimiento escolar? Valoras. UC, 1-8. Recuperado de http://valoras.uc.cl/wp-content/uploads/2010/10/ factores_familia.pdf

Sameroff, A. J. y Mackenzie, M. J. (2003). Research strategies for capturing transactional models of development: The limits of the possible. Development and Psychopathology, 15(3), 613-640.

Solís-Cámara, P. y Díaz, M. (2007). Relaciones entre creencias y prácticas de crianza de padres con niños pequeños. Anales de Psicología, 23(2), 177-184.

Spano, S. (2004). Stages of adolescent development. Act for Youth Upstate Center of Excellence. Recuperado de http//: actforyouth.net/ resources/rf/rf_stages_0504.pdf

Sroufe, A. (2009). The concept of development in developmental psychopathology. Child Development Perspectives, 3(3), 178-183. Recuperado de http://doi.org/10.1111/j.1750-8606.2009.00103.x

UNICEF (2004). Participación de las familias en la educación infantil latinoamericana. Chile: UNICEF.

(2011). La adolescencia. Una etapa de oportunidades. Nueva York: UNICEF. 